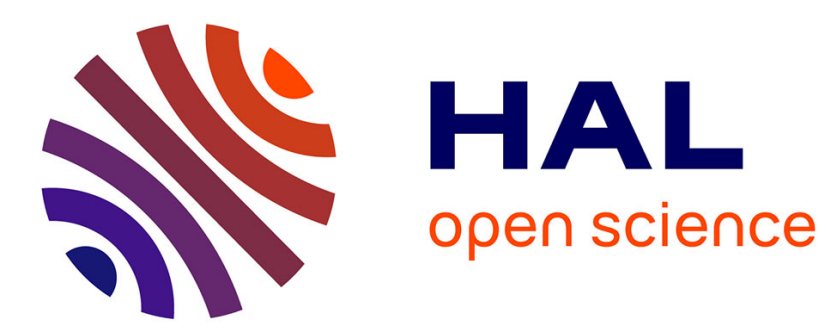

\title{
Strange perspectives at FAIR
}

J Steinheimer, C Sturm, S Schramm, H Stöcker

\section{To cite this version:}

J Steinheimer, C Sturm, S Schramm, H Stöcker. Strange perspectives at FAIR. Journal of Physics G: Nuclear and Particle Physics, 2010, 37 (9), pp.94026. 10.1088/0954-3899/37/9/094026 . hal00600845

\section{HAL Id: hal-00600845 https://hal.science/hal-00600845}

Submitted on 16 Jun 2011

HAL is a multi-disciplinary open access archive for the deposit and dissemination of scientific research documents, whether they are published or not. The documents may come from teaching and research institutions in France or abroad, or from public or private research centers.
L'archive ouverte pluridisciplinaire HAL, est destinée au dépôt et à la diffusion de documents scientifiques de niveau recherche, publiés ou non, émanant des établissements d'enseignement et de recherche français ou étrangers, des laboratoires publics ou privés. 


\title{
Strange Perspectives at FAIR
}

\author{
J. Steinheimer ${ }^{1}$, C. Sturm ${ }^{2,3}$, S. Schramm ${ }^{4,5}$ and H. Stöcker ${ }^{1,3,5}$ \\ 1 Institut für Theoretische Physik, Goethe Universität Frankfurt, \\ Max-von-Laue Str. 1, D-60438 Frankfurt Main, Germany \\ 2 Institut für Kernphysik, Goethe Universität Frankfurt, \\ Max-von-Laue Str. 1, D-60438 Frankfurt Main, Germany \\ 3 GSI Helmholtzzentrum für Schwerionenforschung GmbH, \\ Planckstr. 1, D-64291 Darmstadt, Germany \\ 4 Center for Scientific Computing, Max-von-Laue-Str. 1, \\ D-60438 Frankfurt am Main, Germany \\ 5 Frankfurt Institute for Advanced Studies, Ruth-Moufang-Str. 1, \\ D-60438 Frankfurt am Main, Germany \\ E-mail: steinheimer@th.physik.uni-frankfurt.de
}

\begin{abstract}
Adjacent to the existing accelarator complex of the GSI Helmholtz Centre for Heavy Ion Research at Darmstadt/Germany the Facility for Antiproton and Ion Research FAIR substantially expands research goals and technical possibilities. It will provide worldwide unique accelerator and experimental facilities allowing for a large variety of unprecedented fore-front research in hadron, nuclear and atomic physics as well as applied sciences which will be described in this article briefly. The start version of FAIR, the so called Modularized Start Version will deliver first beams in 2015/2016. As an example the article presents research efforts on strangeness at FAIR using heavy ion collisions, exotic nuclei from fragmentation and antiprotons to tackle various topics in this area. In particular hypernuclei and metastable exotic multihypernuclear objects will be investigated.
\end{abstract}




\section{The FAIR Project}

The Facility for Antiproton and Ion Research, FAIR [1, 2, 3], will provide an extensive range of particle beams from protons and antiprotons to ion beams of all chemical elements up to the heaviest one, uranium, with in many respects world record intensities. As a joint effort of 16 countries $\ddagger$ the new facility builds, and substantially expands, on the present accelerator system at GSI, both in its research goals and its technical possibilities. Compared to the present GSI facility, an increase of a factor of 100 in primary beam intensities, and up to a factor of 10000 in secondary radioactive beam intensities, will be a technical property of the new facility.

The start version of FAIR, the so called Modularized Start Version includes a basic accelerator as well as three experimental modules as it is colored illustrated in figure 1. The superconducting synchrotron SIS100 with a circumference of 1100 meters and a magnetic rigidity of $100 \mathrm{Tm}$ is at the heart of the FAIR accelerator facility. Following an upgrade for high intensities, the existing GSI accelerators UNILAC and SIS18 will serve as an injector. Adjacent to the SIS100 synchrotron are two storage-cooler rings and experiment stations, including a superconducting nuclear fragment separator (SuperFRS) and an antiproton production target. By delivering first beams FAIR will supply rare isotope beams (RIBs) and antiproton beams with excellent intensity and quality.

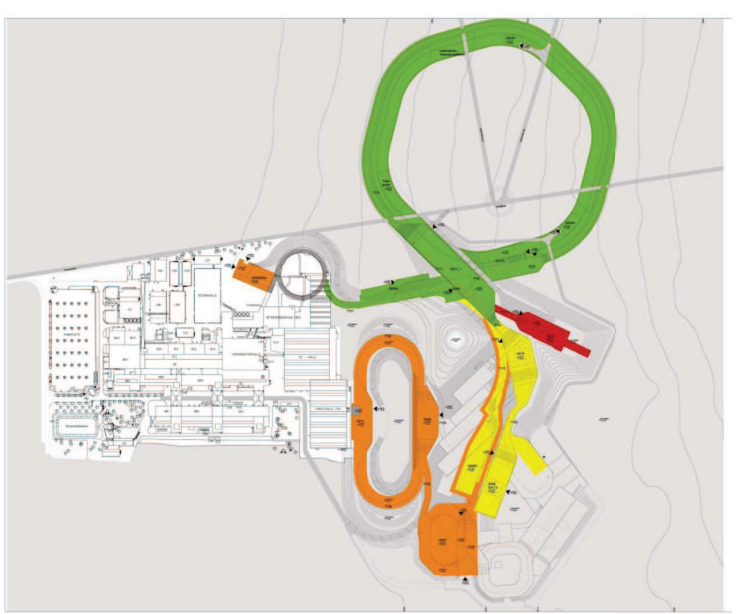

Figure 1. On the left hand side the existing GSI facility is shown. Colored displayed is the so called Modularized Start Version of FAIR including module 0 - 3 . Coloring: the $100 \mathrm{Tm}$ super conducting synchrotron SIS100 (module 0) - green; the experimental area for CBM/HADES (module 1) - red; the NuSTAR facility including the Super-FRS (module 2) yellow; The Antiproton facility including the PANDA experiment (module 3) orange. Not shown is the additional experimental area above ground for the APPA community (module 1).

Because of the long lead times for civil-construction planning civil work for the first buildings of FAIR will start in the fourth quarter of 2010 earliest. First beams will be delivered in 2015/2016. Moreover, after the start phase the facility will be upgraded by experimental storage rings enhancing capabilities of secondary beams and by the SIS300 providing particle energies 20-fold higher compared to those achieved so far at GSI as additional funds become available.

‡ In alphabetical order: Austria, China, Finland, France, Germany, Greece, India, Italy, Poland, Romania, Russia, Slovenia, Slovakia, Spain, Sweden, and the United Kingdom 


\section{The Experimental Programme of FAIR}

The main thrust of FAIR research focuses on the structure and evolution of matter on both a microscopic and on a cosmic scale. The approved FAIR research programme embraces 14 experiments, which form the four scientific pillars of FAIR and offers a large variety of unprecedented forefront research in hadron, nuclear, atomic and plasma physics as well as applied sciences. Already today, over 2500 scientists and engineers are involved in the design and preparation of the FAIR experiments. They are organized in the experimental collaborations APPA, CBM, NuSTAR, and PANDA.

\subsection{APPA - Atomic Physics, Plasma Physics and Applications}

Atomic physics with highly charged ions [4] will concentrate on two central research themes: (i) the correlated electron dynamics in strong, ultra-short electromagnetic fields including the production of electron-positron pairs and (ii) fundamental interactions between electrons and heavy nuclei in particular the interactions described by Quantum Electrodynamics, QED. Here bound-state QED in critical and supercritical fields is the focus of the research programme. In addition, atomic physics techniques will be used to determine properties of stable and unstable nuclei and to perform tests of predictions of fundamental theories besides QED.

For Plasma physics the availability of high-energy, high-intensity ion-beams enables the investigation of High Energy Density Matter in regimes of temperature, density and pressure not accessible so far [5]. It will allow probing new areas in the phase diagram and long-standing open questions of basic equation of state research can be addressed. The biological effectiveness of high energy and high intensity beams was never studied in the past. It will afford to investigate the radiation damage induced by cosmic rays and protection issues for the Moon and Mars missions. Furthermore, the intense ion-matter interactions with projectiles of energies above $1 \mathrm{GeV} / \mathrm{u}$ will endorse systematic studies of material modifications.

\section{2. $C B M / H A D E S$ - Compressed Baryonic Matter}

Violent collisions between heavy nuclei promise insight into an unusual state in nature, that of highly compressed nuclear matter. In addition to its relevance for understanding fundamental aspects of the strong interaction, this form of matter may exist in various so far unexplored phases in the interior of neutron stars and in the core of supernovae. HADES $[6,7,8]$ and CBM $[9,10]$ at SIS100/300 will explore the QCD phase diagram in the region of very high baryon densities and moderate temperatures. This approach includes the study of the nuclear matter equation-of-state, the search for new forms of matter, the search for the predicted first order phase transition between hadronic and partonic matter, the QCD critical endpoint, and the chiral phase transition, which is related to the origin of hadron masses. It is intended to perform comprehensive measurements of hadrons, electrons, muons and photons created in collisions of heavy 
nuclei proton-nucleus, and proton-proton collisions at different beam energies. Most of the rare probes like lepton pairs, multi-strange hyperons and charm will be measured for the first time in the FAIR energy range.

\subsection{NuSTAR - Nuclear Structure, Astrophysics and Reactions}

The main scientific thrusts in the study of nuclei far from stability are aimed at three areas of research: (i) the structure of nuclei, the quantal many-body systems built by protons and neutrons and governed by the strong force, towards the limits of stability, where nuclei become unbound, (ii) nuclear astrophysics delineating the detailed paths of element formation in stars and explosive nucleosynthesis that involve short-lived nuclei, (iii) and the study of fundamental interactions and symmetries exploiting the properties of specific radioactive nuclei.

The central part of the NuSTAR programme at FAIR $[11,12]$ is the high acceptance Super-FRS with its multi-stage separation that will provide high intensity mono-isotopic radioactive ion beams of bare and highly-ionized exotic nuclei at and close to the driplines. This separator, in conjunction with high intensity primary beams with energies up to $1.5 \mathrm{AGeV}$, is the keystone for a competitive NuSTAR physics programme. This opens the unique opportunity to study the evolution of nuclear structure into the yet unexplored territory of the nuclear chart and to determine the properties of many short-lived nuclei which are produced in explosive astrophysical events and crucially influence their dynamics and associated nucleosynthesis processes.

\subsection{PANDA - AntiProton ANnihilation in DArmstadt}

The big challenge in hadron physics is to achieve a quantitative understanding of strongly interacting complex systems at the level of quarks and gluons. In $p \bar{p}$-annihilation, particles with gluonic degrees of freedom as well as particle-antiparticle pairs are copiously produced, allowing spectroscopic studies with unprecedented statistics and precision. The PANDA experiment at FAIR [13, 14] will bring new fundamental knowledge in hadron physics by pushing the precision barrier towards new limits. The charmonium $(c \bar{c})$ spectroscopy will take advantage by precision measurements of mass, width, decay branches of all charmonium states. Particular emphasis is placed on mesons with open and hidden charm, which extends ongoing studies in the light quark sector to heavy quarks, and adds information on contributions of the gluon dynamics to hadron masses. The search for exotic hadronic matter such as hybrid mesons or heavy glueballs gains enormously by precise scanning of resonance curves of narrow states as well. Recently, this field has attracted much attention with the surprise observation at electron-positron colliders of the new X, Y and Z states with masses around $4 \mathrm{GeV}$. These heavy particles show very unusual properties, whose theoretical interpretation is entirely open. Additionally the precision gamma-ray spectroscopy of single and double hypernuclei will allow extracting information on their structure and on the hyperonnucleon and hyperon-hyperon interaction. 


\section{Strangeness physics at FAIR}

Massive nucleus-nucleus collisions provide an abundant source of strangeness: more than 50 Hyperons and about 30 Anti-Kaons are produced in central collisions of lead nuclei at the CERN-SPS low energy programme and before that at the AGS. With its high luminosity beam the FAIR facility and the CBM experiment offer the opportunity to do high precision measurements of bulk observables related to strangeness production (e.g. ratios, spectra and fluctuations). In addition it will open the door to explore the 3rd dimension of the nuclear chart, as hypernuclei and other exotic forms of bound objects containing strangeness will be produced abundantly at the CBM, PANDA and NuSTAR experiments.

\subsection{Exotic hypermatter}

These forms of metastable or even stable objects with strangeness have been proposed long ago (see [15]) as collapsed states of matter, either consisting of baryons or quarks. For example the $\mathrm{H}$ di-baryon (a six quark state) was predicted by Jaffe [16]. The (non)observation of multi-quark bags, e.g. strangelets and (strange) di-baryons is still one of the open problems of intermediate and high energy physics.

Recent calculations within an effective chiral mean field model for infinite matter $[17,18]$ predict that stable and metastable strange matter can be created when the net strangeness of the bulk matter becomes larger than $f_{s}>0.4$. In this simple estimate we set the strength of the hyperon coupling to the strange scalar $\zeta$, whose mean-field value corresponds to the scalar strange-quark condensate, to a value of $n_{s} g_{2}\left(n_{s}\right.$ is the number of strange quarks in the hyperon), rescaling the other couplings to keep the correct vacuum masses of the particles. The full line in figure 2 (left) shows the corresponding
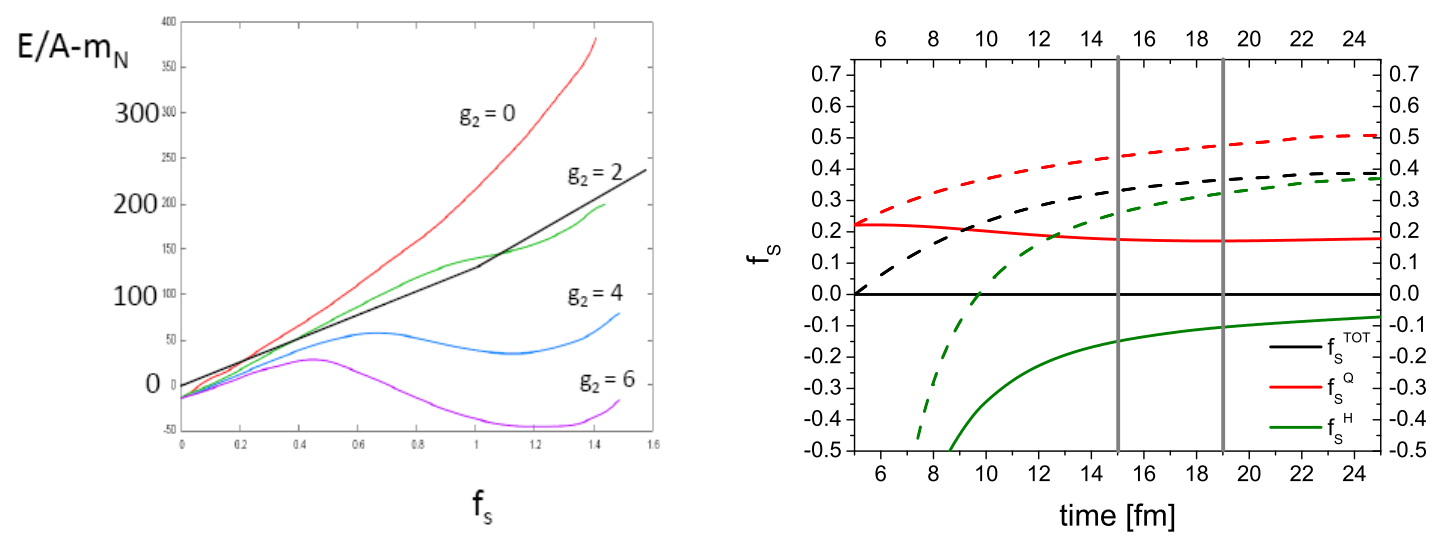

Figure 2. Left: Binding energy per particle as a function of strangeness per baryon $f_{s}$ for different values of the hyperon interaction with the scalar field $\zeta\left(g_{2}\right)$. Right: Time evolution of the strangeness per baryon fraction for the 2 coexisting phases (hadrons or quarks) in a heavy ion collision at $E_{l a b}=40 \mathrm{~A} \mathrm{GeV}$. The solid line corresponds to results where the fireball has vanishing net strangeness while the dashed line shows results where emission of Kaons is included. 
values of lowest energy of a system of free nucleons and hyperons for a given $f_{s}$ (net strangeness per baryon). Beyond a value of $g_{2} \approx 2$ one obtains a bound state for fixed $f_{s}$. For even higher values of the coupling strength absolutely stable hypermatter at $f_{s} \approx 1.2$ is obtained.

It was shown, within a hadronic transport model (UrQMD) [19], that strangeness can be separated in phase space due to the non-equilibrium nature of the early phase of a heavy ion collision. Furthermore, in equilibrated fireballs, strange and anti-strange quarks separate by a distillation process. While strangeness is produced exclusively in pairs in the quark gluon plasma phase, hadronic production involves mostly the associated production of a $K^{+}$and $\Lambda$ hyperon, which leads to a chemical imbalance if both phases coexist. To regain chemical phase equilibrium the quark phase of the fireball will be loaded with net strangeness.

In addition the emission of Kaons can further enrich the produced fireball with strange quarks. For our calculations we apply an equation of state that follows from an effective chiral $\mathrm{SU}(3)_{f}$ Lagrangian, including the full sets of mesonic and baryonic octets. Quark and gluonic degrees of freedom are included to model the deconfinement phase transition [20]. Both, chiral symmetry restoration and deconfinement, are described as a smooth crossover in the model. As a result we observe a wide region of phase coexistence in the phase diagram, which gives room for a possible distillation scenario.

Figure 2 (right) shows the time evolution (in the center of mass frame of the collision) of the strangeness per baryon fraction for a fireball that is created at a $\mathrm{Pb}+\mathrm{Pb}$ collision at $E_{l a b}=40 \mathrm{~A} \mathrm{GeV}$. The two vertical lines set the limits to where one would expect a chemical freeze out to occur. Beyond this time, the system decouples and should not be treated as being in chemical equilibrium. The solid lines depict the strangeness fraction in the different phases, while the fireball has no net strange charge. This reflects the original strangeness distillation process as proposed in [21]. In our model this process will lead to a strangeness fraction of $f_{s} \approx 0.2$ of the remaining quark droplet, at freeze out.

Due to early emission of Kaons the strangeness fraction can even increase more during the course of the fireballs evolution, as Kaons are more abundant at hight baryon densities than Antikaons [22]. The fireball itself becomes loaded with net strangeness. In combination with the distillation process, the quark part of the system can obtain a strangeness fraction of up to $f_{s} \approx 0.5$. This could in turn result in the formation of a (meta-)stable cluster of quarks or hadrons.

\subsection{Hypernuclei}

Conventional hypernuclei are known to exist already for a long time [24, 25, 26]. At the future FAIR facility several experiments will perform ambitious research programmes in hypernuclear physics. At the PANDA experiment for example, precision gamma-ray spectroscopy of single and double hypernuclei will be performed to investigate their structure, the hyperon-nucleon and hyperon-hyperon interaction as well as the weak 
decays of these objects.

As the FAIR accelerator offers an unprecedented beam intensity for heavy ion experiments, hypernuclei and multi-hypernuclear objects will be produced abundantly in the CBM experiment [20]. Here the strange matter clusters are created from a hot and dense fireball. In the following we will discuss the production of the single $\Lambda$ Hypernucleus from thermal equilibrium. More precisely we will calculate the following ratio $R_{H}$

$$
R_{H}={ }_{\Lambda}^{3} H /{ }^{3} \mathrm{He} \cdot p / \Lambda
$$

for collisions of $\mathrm{Pb}+\mathrm{Pb}$ at a wide range of beam energies. This ratio is especially interesting, as it does not depend on the chemical potential of the particles (as fugacities cancel), and any canonical correction factors for strangeness are canceled.

First we calculate the ratio in a simple thermal model, where we assume a single freeze out temperature for every beam energy. In our calculation we adapted the freeze out curve which was found in a thermal model analysis of Andronic et. al. [27]. The resulting excitation function is shown in figure 3 as dotted line. It is obvious that the temperature dependence of the proposed ratio is (almost) negligible. Please note that, for simplicity, we have neglected the contribution of the feed down from higher resonances to the $\Lambda$ and proton yields. This contribution will be discussed later.

To get a more sophisticated picture of thermal production we also calculate $R_{H}$ from a hybrid model simulation of heavy ion collisions. In this model a full $3+1$ dimensional ideal hydrodynamic evolution is embedded in the UrQMD transport code. At the transition hypersurface from hydrodynamics to transport all particles are thermally produced according to the Cooper Frye prescription [28].

The solid line in figure 3 shows results on $R_{H}$ from the hybrid model, extracted at the transition surface from hydrodynamics to transport. When final state interactions (FSI), like resonance decays and production, are explicitly included in the transport phase, the result changes significantly (dashed line). Nevertheless it still does not depend

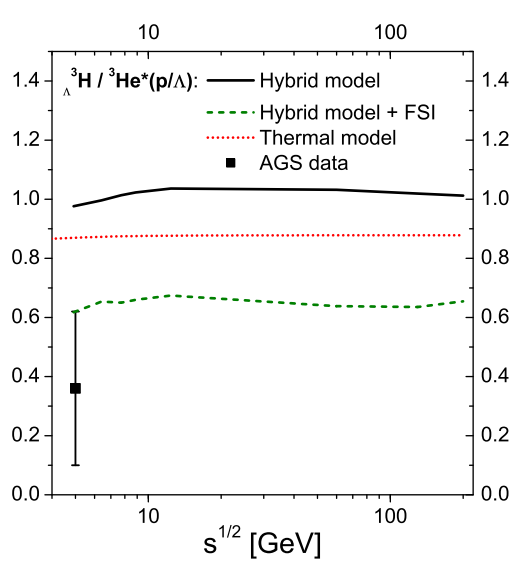

Figure 3. Excitation function of the ratio $R_{H}$ (see text) for different model calculations compared to data [23]. 
on the beam energy. Note that we neglected contributions to ${ }_{\Lambda}^{3} H /{ }^{3} H e$ from FSI (e.g. via coalescence of decay products) which should again increase $R_{H}$.

Comparing our different results to data taken at the AGS [23], a large discrepancy can be seen, while preliminary results from RHIC experiment are in line with predictions from thermal production $\left(R_{H} \approx 1\right)$ [29]. This discrepancy can be explained by the non equilibrium (not canonical) nature of strangeness production at AGS energies. The FAIR facility therefore offers the opportunity to study the production of such hypernuclei over a very interesting range of beam energies. It may prove to be a unique tool to study the process of strangeness equilibration which might be closely related to the formation of a QGP [30, 31, 32].

\section{Summary}

FAIR will allow forefront research addressing (1) the investigation of the properties and the role of the strong (nuclear) force in shaping basic building blocks of the visible world around us and of its role in the evolution of the universe, (2) tests of symmetries and predictions of the standard model and search for physics beyond in the electro-weak sector and in the domain of the strong interaction, (3) the properties of matter under extreme conditions, both at the subatomic as well as at the macroscopic scale of matter and (4) applications of high-intensity, high-quality ion and antiproton beams in research areas that provide the basis for, or directly address, issues of applied sciences and technology. Due to the high luminosity which exceeds current facilities by up to a factor of 10000, experiments will be feasible that could not be done elsewhere. This opens the unique opportunity to study the evolution of nuclear structure into the yet unexplored 3rd dimension of the nuclear chart and to determine the properties of many short-lived hypernuclei and metastable exotic multi-hypernuclear objects. FAIR will expand the knowledge in the various scientific fields beyond current frontiers. Moreover, there exists strong cross-topical synergies which can be exploited and promise novel insights.

\section{Acknowledgments}

This work was supported by HGS-HIRe and the Hessian LOEWE initiative through the Helmholtz International Center for FAIR (HIC for FAIR). The computational resources were provided by the Frankfurt Center for Scientific Computing (CSC).

\section{References}

[1] H.H. Gutbrod et al. (Eds.) FAIR Baseline Technical Report, ISBN 3-9811298-0-6 Nov. 2006.

[2] W. F. Henning, FAIR: Recent developments and status, Nucl. Phys. A 805 (2008) 502.

[3] H. Stöcker, FAIR: Challenges Overcome and Still to be Met, published in Conf.Proc.C0806233:moycgm01, 2008.

[4] Th. Stöhlker et al., Atomic physics with highly-charged ions at the future FAIR facility: A status report, Nuclear Instruments and Methods in Physics Research B 261 (2007) 234-238. 
[5] I.V. Lomonosov and N.A. Tahir, Prospects of High-Energy Density Matter Research at the Future FAIR Facility at Darmstadt, Nuclear Physics News 16 (1) (2006) 29 - 35.

[6] G. Agakishiev et al. [HADES Collaboration], The High-Acceptance Dielectron Spectrometer HADES, Eur. Phys. J. A 41, 243 (2009)

[7] J. Stroth et al. [HADES Collaboration], Di-electron emission from resonance matter, Prog. Part. Nucl. Phys. 62 (2009) 481.

[8] I. Fröhlich for the HADES Collaboration, Future perspectives at SIS-100 with HADES-atFAIR, arXiv:0906.0091 [nucl-ex].

[9] P. Senger et al. , Compressed baryonic matter: Experiments at GSI and FAIR, Phys. Part. Nucl. 39 (2008) 1055.

[10] P. Senger et al. , Probing dense baryonic matter, Prog. Part. Nucl. Phys. 62 (2009) 375.

[11] R. Krücken [NuSTAR Collaboration], The Nustar Facility At Fair, J. Phys. G 31 (2005) S1807.

[12] B. Rubio and T. Nilsson, Nustar, Nucl. Phys. News 16 (2006) 9.

[13] K. Fohl et al., The Panda Detector At The Future Fair Laboratory, Eur. Phys. J. ST 162 (2008) 213 .

[14] J. S. Lange for the PANDA Collaboration, The Panda Experiment: Hadron Physics With Antiprotons At Fair, Int. J. Mod. Phys. A 24 (2009) 369.

[15] A. R. Bodmer, Phys. Rev. D 4, 1601 (1971).

[16] R. L. Jaffe, Phys. Rev. Lett. 38, 195 (1977)

[17] P. Papazoglou, D. Zschiesche, S. Schramm, J. Schaffner-Bielich, H. Stoecker and W. Greiner, Phys. Rev. C 59, 411 (1999)

[18] P. Papazoglou, S. Schramm, J. Schaffner-Bielich, H. Stoecker and W. Greiner, Phys. Rev. C 57, 2576 (1998)

[19] J. Steinheimer, M. Mitrovski, T. Schuster, H. Petersen, M. Bleicher and H. Stoecker, Phys. Lett. B 676, 126 (2009)

[20] J. Steinheimer, S. Schramm and H. Stocker, arXiv:0909.4421 [hep-ph].

[21] C. Greiner, P. Koch and H. Stoecker, Phys. Rev. Lett. 58, 1825 (1987).

[22] C. Greiner, D. H. Rischke, H. Stoecker and P. Koch, Phys. Rev. D 38, 2797 (1988).

[23] T. A. Armstrong et al. [E864 Collaboration], Phys. Rev. C 70, 024902 (2004)

[24] J. K. Ahn et al., Phys. Rev. Lett. 87, 132504 (2001).

[25] H. Takahashi et al., Phys. Rev. Lett. 87, 212502 (2001).

[26] R. H. Dalitz, D. H. Davis, P. H. Fowler, A. Montwill, J. Pniewski and J. A. Zakrzewski, Proc. Roy. Soc. Lond. A 426, 1 (1989)

[27] A. Andronic, P. Braun-Munzinger and J. Stachel, Phys. Lett. B 673, 142 (2009)

[28] H. Petersen, J. Steinheimer, G. Burau, M. Bleicher and H. Stocker, Phys. Rev. C 78, 044901 (2008)

[29] J. H. Chen and f. t. S. Collaboration, Nucl. Phys. A 830, 761C (2009)

[30] P. Koch, B. Muller and J. Rafelski, Phys. Rept. 142, 167 (1986).

[31] S. Soff et al., Phys. Lett. B 471, 89 (1999)

[32] C. Greiner, P. Koch-Steinheimer, F. M. Liu, I. A. Shovkovy and H. Stoecker, J. Phys. G 31, S725 (2005) 